\title{
Increased in vivo immunological potency of HB-110, a novel therapeutic HBV DNA vaccine, by electroporation
}

\author{
Chae Young Kim ${ }^{1,3}$, Eun Sung Kang ${ }^{1}$, \\ Seon Beom Kim ${ }^{1}$, Han Eol Kim ${ }^{1}$, Jae Hoon Choi ${ }^{1}$, \\ Dong Sop Lee ${ }^{1}$, Se Jin $\mathrm{Im}^{2}$, Se Hwan Yang ${ }^{2}$, \\ Young Chul Sung ${ }^{2}$, Byong Moon Kim ${ }^{1}$ and \\ Byung-Gee Kim ${ }^{3,4}$ \\ ${ }^{1}$ Research Laboratories \\ Dong-A Pharm. Co., Ltd. \\ Yongin 449-900, Korea \\ ${ }^{2}$ National Research Laboratory of DNA Medicine \\ Division of Molecular and Life Sciences \\ Pohang University of Science and Technology \\ Pohang 790-784, Korea \\ ${ }^{3}$ Interdisciplinary Program of Biochemical Engineering \\ and Biotechnology \\ Seoul National University \\ Seoul 151-742, Korea \\ ${ }^{4}$ Corresponding author: Tel, 82-2-880-6774; \\ Fax, 82-2-874-1206; E-mail, byungkim @ snu.ac.kr \\ DOI 10.3858/emm.2008.40.6.669
}

Accepted 14 August 2008

Abbreviations: DI, direct injection; EP, electorporation; TA, tibialis anterior

\begin{abstract}
Pulse-induced permeabilization of cellular membranes, generally referred to as electroporation (EP), has been used for years as a tool to increase macromolecule uptake in tissues, including nucleic acids, for gene therapeutic applications, and this technique has been shown to result in improved immunogenicity. In this study, we assessed the utility of EP as a tool to improve the efficacy of HB-110, a novel therapeutic DNA vaccine against chronic hepatitis $B$, now in phase 1 of clinical study in South Korea. The potency of HB-110 in mice was shown to be improved by EP. The rapid onset of antigen expression and higher magnitude of humoral and cellular responses in electric pulse-treated mice revealed that EP may enable a substantial reduction in the dosage of DNA vaccine required to elicit a response similar in magnitude to that achievable via conventional administration. This study also showed that EP-based vaccination at 4-week-intervals elicited a cellular immune response which was about two-fold
\end{abstract}

higher than the response elicited by conventional vaccination at 2-week intervals. These results may provide a rationale to reduce the clinical dose and increase the interval between the doses in the multidose vaccination schedule. Electric pulsing also elicited a more balanced immune response against four antigens expressed by HB-110: S, preS, Core, and Pol.

Keywords: electroporation; hepatitis B virus; vaccination; vaccines, DNA

\section{Introduction}

Remarkable progress has been made in gene transfer technology, which confers upon "genes" the potential to substitute for classic protein vaccines as preventive or therapeutic vaccines. An ideal gene delivery system should possess well-characterized pharmaceutical properties; it should provide prolonged expression of transgenes at therapeutic levels, with the ability to be re-dosed; it should not induce auto-antibody responses; and it should be able to be manufactured at a reasonable cost. Plasmid DNA fulfills all of these requirements, but tends to be ineffective as the result of its poor cellular uptake. There are a number of strategies by which the cellular uptake of plasmid DNA might be increased, including formulation (O'Hagan et al., 2004; Otten et al., 2006), genetic adjuvant coadministration (Calarota et al., 2004; Otten et al., 2006), and physical methods, such as in vivo electroporation (Andre et al., 2004; Otten et al., 2004, 2006; Heller et al., 2006). The application of electrical pulses (electroporation) has been demonstrated to overcome this fatal flaw of plasmid DNA by increasing its cellular permeability, which results in an increase of cellular DNA uptake, high level protein expression, and ultimately, improved humoral and cellular immune responses (Hurk et al., 2004; Wang et al., 2005; Luzembourg, 2006; Tjelle et al., 2006; Penga et al., 2007). It has been reported that the magnitude of immune responses induced in primates is lower than that in small animals, and the quantity of DNA required for the effective immunization of primates is significantly higher (mg vs $\mu \mathrm{g}$ ) (Aihara et al., 1998; Selby et al., 2000). Electroporation (EP) resolves this problem via the introduction of plasmid DNA into the cells to a degree adequate for optimal immune response at 
low dosages (Luckay et al., 2007).

The first clinical trial of electroporation in a gene delivery protocol is currently in phase 1 , and is being spearheaded by the Moffit Cancer Center using plasmid DNA encoding for IL-12 against malignant melanoma. Positive interim results were reported at the 9th annual meeting of the American Society of Gene Therapy (ASGT). Additionally, five phase 1 studies of electroporation for gene delivery are currently underway, and deal with therapies against malignant melanoma (Vical, and Ichor Medical system), breast, ovarian, and colorectal cancers (Merck\&Co), prostate cancer (University of Southhampton), and HCV (Tripep AB). Many clinical and preclinical studies have demonstrated that the application of electroporation is safe and well tolerated in primates, including humans, and are, moreover, very effective in enabling the plasmid DNA to elicit an immune response. In this study, we evaluated the utility of electroporation as a tool to improve the efficacy of HB-110, a novel therapeutic DNA vaccine against chronic hepatitis $B$, currently in phase 1 of clinical study in South Korea.

\section{Materials and Methods}

\section{Plasmid preparation}

HB-110 consists of three plasmids, pGX10-S/L, pGX10-C/P, and pGX10-hIL-12m, which encode for the HBV envelope proteins $(S, L)$, core protein, polymerase, and human IL-12, respectively. They were formulated in $150 \mathrm{mM}$ phosphate buffer, at neutral $\mathrm{pH}$, at a ratio of $2: 1: 1$. The plasmid vectors were constructed via the cloning of the respective genes into the eukaryotic expression vector, pGX10. The pGX10 backbone harbors a prokaryotic origin of replication (ColE1), a bacterial kanamycin resistance gene, and a eukaryotic expression cassette consisting of the human CMV promoter, adenovirus tripartite leader sequence, SV40 late polyA, and SV40 enhancer. Each plasmid was generated in $E$. coli $\mathrm{DH} 5 \alpha$ cells using a $15 \mathrm{~L}$ fermentor. The fermentation broth was then subjected to a series of purification steps including alkaline lysis, PEG precipitation, anion exchange chromatography, and thiophilic/aromatic adsorption chromatography. The purified plasmids were mixed to a final composition and dialyzed against the phosphate buffer.

\section{Experimental animals and immunization}

Male 6-week-old Balb/c mice received $100 \mu \mathrm{g}$ of total plasmid DNA (in a volume of $50 \mu \mathrm{l}$ ) on the left tibialis anterior (TA) muscle, immediately followed by electroporation. Two needle array electrodes (BTX ECM 830) were utilized for electroporation. The distance between the electrodes was $5 \mathrm{~mm}$, and the array was inserted longitudinally into the muscle fibers. In vivo electroporation was conducted at $100 \mathrm{~V} / \mathrm{cm}$ using a BTX 830 wave generator. 6 unipolar pulses were applied, each lasting for 20 milliseconds. Immunizations were conducted three times at weeks 0,2 , and 4 or twice at weeks 0 and 4 for the evaluation of immune response. Antigen expression was assessed after the administration of a single dose.

\section{Measurement of expressed S antigen}

Mice were sacrificed on days 2, 5, 10, and 20 after vaccination, and the anterior tibialis muscles were removed and stored in a deep freezer until analysis. 5 mice were used per group. The frozen tissue was homogenized with a OMNI homogenizer (OMNI international, TH-115, San Diego, CA) in $400 u$ of lysis buffer $(25 \mathrm{mM}$ Tris, $\mathrm{pH} 7.4,50$ $\mathrm{mM} \mathrm{NaCl}, 0.5 \% \mathrm{Na}$-Doxycholate, $2 \% \mathrm{NP}-40,0.2 \%$ SDS) containing protease inhibitor cocktail (Promega, Madison, $\mathrm{WI}$ ), and was then subjected to an HBs ELISA assay (ETI- MAK-4, N0019, DiaSorin, Saluggia, Italy).

\section{Measurement of antibody responses}

Mice vaccinated twice at 4-week intervals were sacrificed on weeks $3,4,6$, and 12 after the second immunization. 5 mice were used per group. Antibody responses against $\mathrm{HBs}$ antigen and $\mathrm{HBC}$ antigen were assessed using anti-HBs ELISA (Murex anti-HBs, C022K95GB, Abbott) and anti-HBc ELISA (Murex anti-HBc, C04GE65GB, Abbott), respectively. The $\mathrm{OD}$ value was directly proportional to the quantity of anti-HBs antibodies, but was inversely proportional to the quantity of anti-HBc antibodies.

\section{IFN- $\gamma$ ELISPOT assay}

Mice vaccinated twice at 4-week intervals or three times at 2-week intervals were sacrificed 3 weeks after the final immunization. 10 mice were used per group. The splenocytes were isolated using ficolle gradients and were subjected to ELISPOT assay using a commercial kit (Mouse IFN- $\gamma$, EL485, R\&D Systems, Minneapolis, MN). The numbers of IFN- $\gamma$ secreting cells were counted with a Bioreader-4000 system (Biosys, Germany). The splenocytes were stimulated by four peptide pools of $S$, preS, Core, and Pol. 


\section{HBsAg seroconversion analysis}

HBV transgenic mice were used which replicate the full HBV genome in the liver. The transgenic mice, originally constructed at Kumamoto University of Japan (Araki et al., 1989), were bred and maintained at Pohang University of Science and Technology. The mice were divided into three groups, receiving either vector control (pGX10), HB-110 or HB-110 with subsequent electroporation. Each group contained three female and three male mice, except for the vector control group, which contained two female and two male mice. Mice were vaccinated three times at 2-week intervals, each with a dose of $50 \mu \mathrm{g}$ of HB-110. Electroporation was carried out as described above. Blood was collected at 2 weeks after final vaccination and analyzed for $\mathrm{HBsAg}$ seroconversion by HBsAg ELISA and anti-HBs antibody ELISA.

\section{Results}

\section{HBs antigen expression}

TA muscles were collected on days 2, 5, 10, and 30 after vaccination with a single dose. A control mouse group was administered DNA only, without

\section{A}

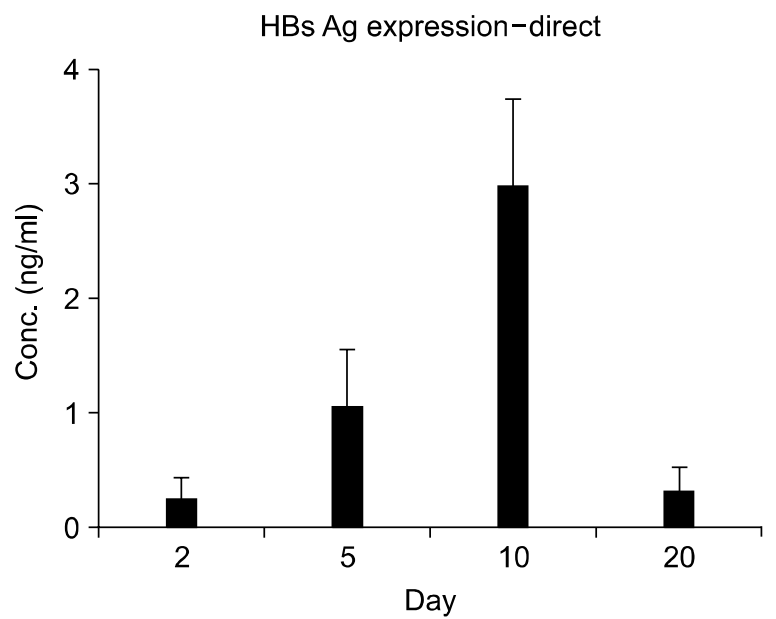

C

\begin{tabular}{r|ccc}
\hline & Direct $(\mathrm{ng} / \mathrm{ml})$ & $E P(\mathrm{ng} / \mathrm{ml})$ & EP/Direct \\
\hline Day 2 & 0.2505 & 23.4388 & $\mathbf{9 3 . 5 5}$ \\
Day 5 & 1.0608 & 90.5755 & $\mathbf{8 5 . 3 8}$ \\
Day 10 & 2.9691 & 99.7986 & $\mathbf{3 3 . 6 1}$ \\
Day 20 & 0.3144 & 10.2302 & $\mathbf{3 2 . 5 4}$ \\
\hline
\end{tabular}

electroporation (referred to as the direct injection group, DI group). In both groups, the peak levels of HBs Ag were achieved on day 10 . However, the electroporation (EP) group evidenced a more rapid increase of antigen expression; the antigen level on day 5 was equivalent to that of day 10 . The increase in expression levels achieved by electroporation was substantial, with the HBsAg levels in the EP group being 30 to 90 -fold higher (Figure 1).

\section{Antibody responses}

The effect of electroporation on antibody response was assessed by measuring the blood level of anti-HBs and anti-HBc on weeks $0,3,4,6,12$. Immunizations were given on weeks 0 and 4 . Humoral immunity was investigated over a time course and with dose escalations (Figure 2, 3). Anti-HBs antibody response was detectable after the 2 nd injection. The EP group evidenced a 3 to 8 -fold enhancement in humoral response as compared to the DI (direct injection) group, and the magnitude of the response increased until week 12 (Figure 2A). Anti-HBc antibody response was detectable after the initial injection and was boosted further after the 2nd injection. Peak $A b$ levels were reached at 6 weeks and were sus-
B

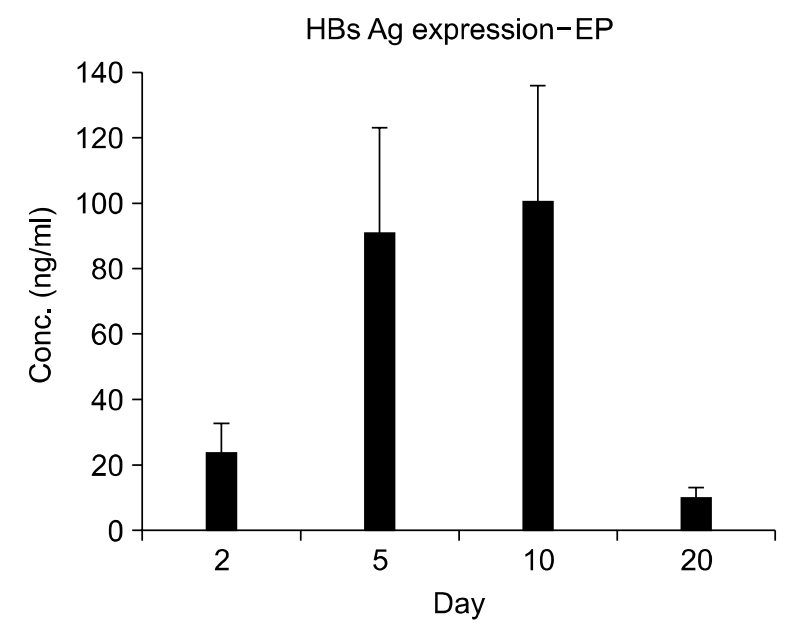

Figure 1. HBsAg expression in TA muscles on days 2, 5, 10, and 30 after vaccination with a single dose. DI group $(A)$ and EP group (B) achieved the peak levels of HBs Ag on day 10 However, the EP group showed a more rapid increase of antigen expression; the antigen level on day 5 was equivalent to that of day 10. The HBsAg levels in the EP group being 30 to 90 -fold higher than those of DI group (C). 
tained until 12 weeks in both groups (Figure $3 \mathrm{~A}$ ) The effect of electroporation on antibody response was less pronounced for $\mathrm{HBc}$, when compared with the results of the anti-HBs. However, the antibody responses were still consistently higher in the EP group.

In the dose escalation study, four dosages (10,

\section{A}

Anti-HBs response-time

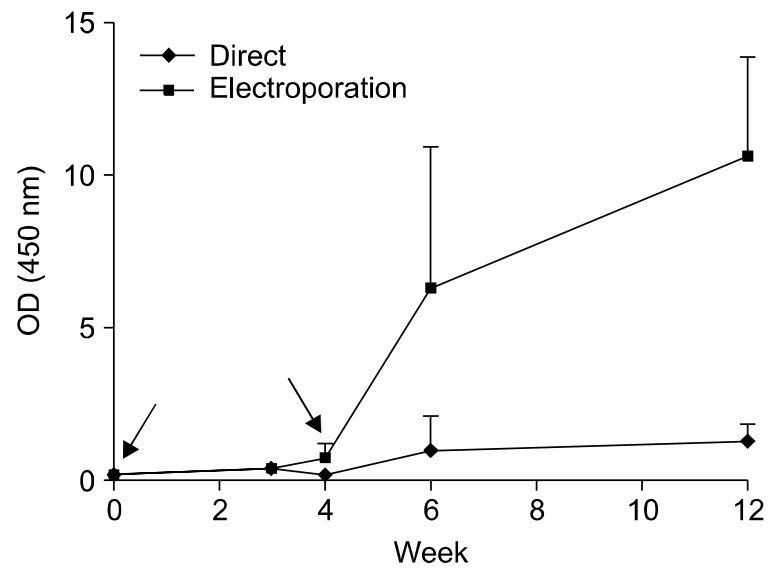

$20,50,100 \mu \mathrm{g}$ of DNA) were evaluated. The titer of anti-HBs antibody in the EP group was increased up to the higher limit of the investigated range, but the DI group evidenced substantially lower responses for all dosages (Figure 2B). However, dose responses of anti-HBc antibody were observed in both the EP and DI groups at the lower ranges of

B

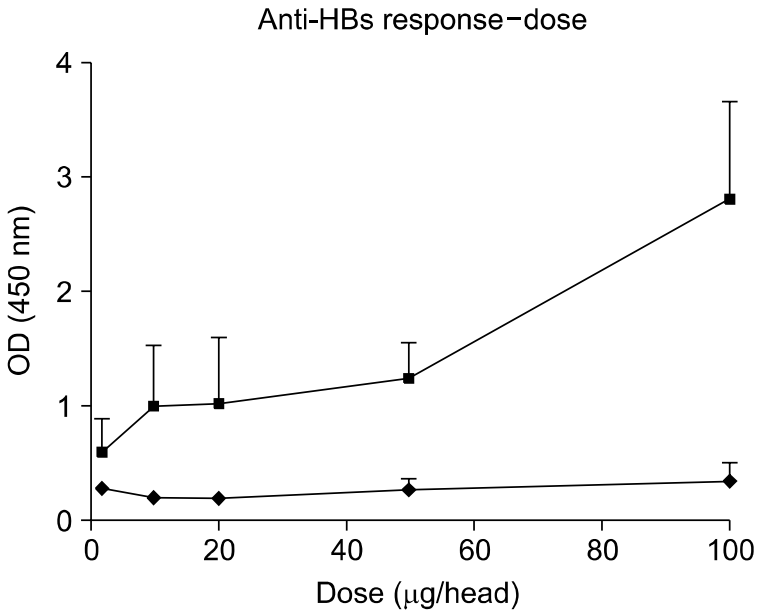

Figure 2. Anti-HBs responses. Mice vaccinated twice at 4-week intervals (depicted as arrows) were sacrificed on weeks 3, 4, 6, and 12. Five mice were used per group. The OD value was directly proportional to the quantity of anti-HBs antibodies. Anti-HBs antibody response was detectable after the $2 \mathrm{nd}$ injection. The EP group showed a 3 to 8 -fold enhancement in humoral response as compared to the DI group, and the magnitude of the response increased until week 12 (A). In the dose escalation study, four dosages $(10,20,50,100 \mu \mathrm{g}$ of DNA) were evaluated on week 12. The titer of anti-HBs antibody in the EP group was increased up to the higher limit of the investigated range, but the DI group evidenced substantially lower responses for all dosages (B).

A

Anti-HBc response-time

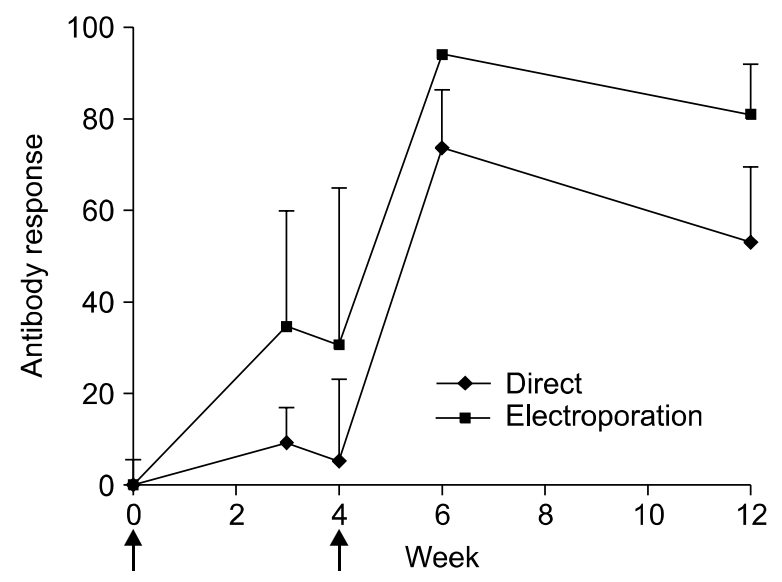

B

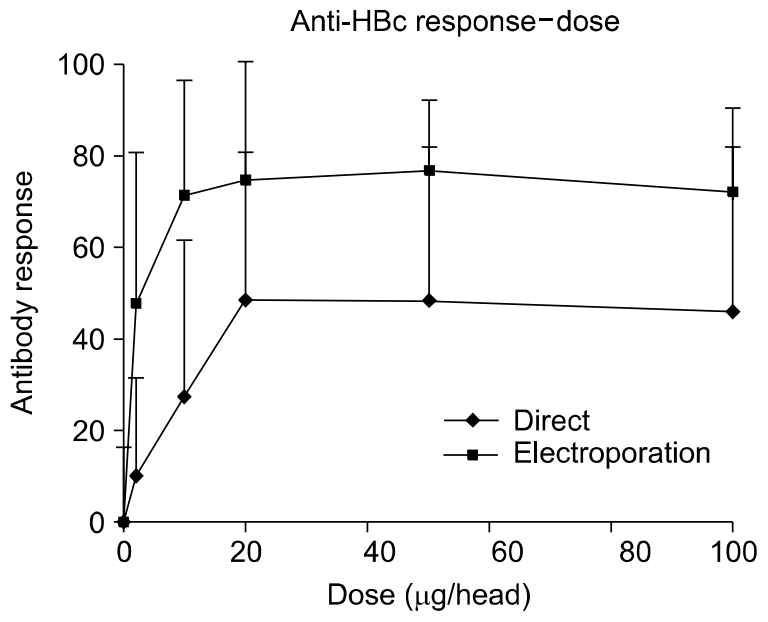

Figure 3. Anti-HBc responses. Mice vaccinated twice at 4-week intervals (depicted as arrows) were sacrificed on weeks $3,4,6$, and 12. Five mice were used per group. Since the assay used to measure antibody response is a competing ELISA, antibody response is defined as $100 \times\left(1-\mathrm{OD}_{\text {sample }} /\right.$ $\mathrm{OD}_{\text {normal plasmal }}$, which is proportional to the amount of anti-HBc antibodies. Anti-HBc antibody response was detectable after the initial injection and was boosted further after the 2 nd injection. Peak Ab levels were reached at 6 weeks and were sustained until 12 weeks in both groups (A). Dose responses of anti-HBc antibody were observed in both the EP and DI groups at the lower ranges of the investigated dosages. The anti-HBc antibody response was saturated at $10 \mu \mathrm{g} /$ head in the EP group and at $20 \mu \mathrm{g} /$ head in the DI group (B). 
the investigated dosages. The anti-HBc antibody response was saturated at $10 \mu \mathrm{g} / \mathrm{head}$ in the EP group and at $20 \mu \mathrm{g} / \mathrm{head}$ in the DI group (Figure 3B).

\section{Cellular immune response}

Cellular immunity is the most important aspect with regard to the determination of the efficacy of a therapeutic DNA vaccine. Thus, cellular immune response was evaluated under several experimental conditions with different numbers and intervals of DNA vaccination. Here, the results of two protocols, three vaccinations at 2-week intervals (protocol 1) and two vaccinations at 4-week intervals (protocol 2), were compared. Cellular immune response was evaluated by counting the number of antigen-specific IFN- $\gamma$ secreting cells. The cellular immunity of HB-110 was enhanced after electric pulse by 1.78 -fold for the $S$ antigen and 3 -fold for other antigens (preS, core, pol) in protocol 1 (3 times/2 wk-interval) (Figure 4A). However, the increase in magnitude due to electroporation was far larger in protocol 2 (twice/4 wk-intervals) than in protocol 1 . In the second protocol, electroporation augmented the immune response by 3 -fold, 13 -fold, 7 -fold, and 11 -fold for $\mathrm{S}$, preS, Core, and Pol, respectively (Figure 4B). The differing potency of electroporation for cellular immunity in the two protocols can be explained as follows. In the DI groups we noted a marked difference between protocol 1 and protocol 2, the former inducing a stronger immune response, presumably as the consequence of the increased immunization frequency (Figure $5 \mathrm{~A}$ ). In the EP groups, the absolute immune response levels were similar for both protocols, thereby indicating that a high level of immune response can be achieved without frequent immunizations (Figure 5B).

Furthermore, electroporation evidenced a broader repertoire of cellular immune responses against all of the antigens, including subdominant antigens such as preS, Core, and Pol, although the magnitude was not as large as with the $S$ antigen.

Electroporation elicited an overall 2-fold increase in cellular immunity in the EP group of protocol 2 (administered two vaccinations at 4-week intervals) as compared to the DI group of protocol 1 (administered three vaccinations at 2-week intervals) (Figure 5C).

\section{HBsAg seroconversion analysis}

Since the appearance of anti-HBs antibodies is a serological marker associated with recovery from natural HBV infection, we investigated whether the humoral response elicited by EP (Figure 2) would be of immunological relevance in a transgenic mouse model of chronic hepatitis B. After three vaccinations of transgenic mice, $50 \%$ of mice receiving vaccine in combination with EP showed HBsAg seroconversion as defined by the absence of HBsAg and the presence of anti-HBs antibody. In contrast, only one case of seroconversion was
A

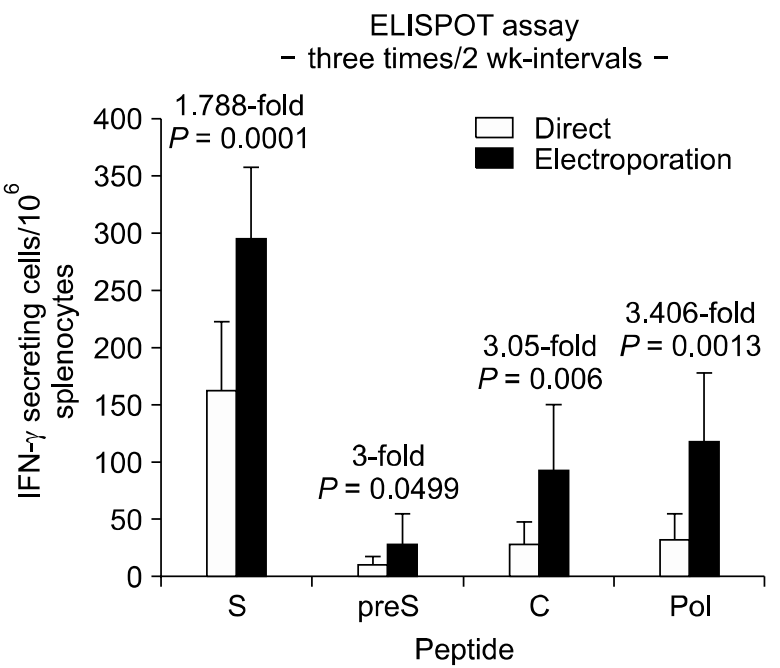

B

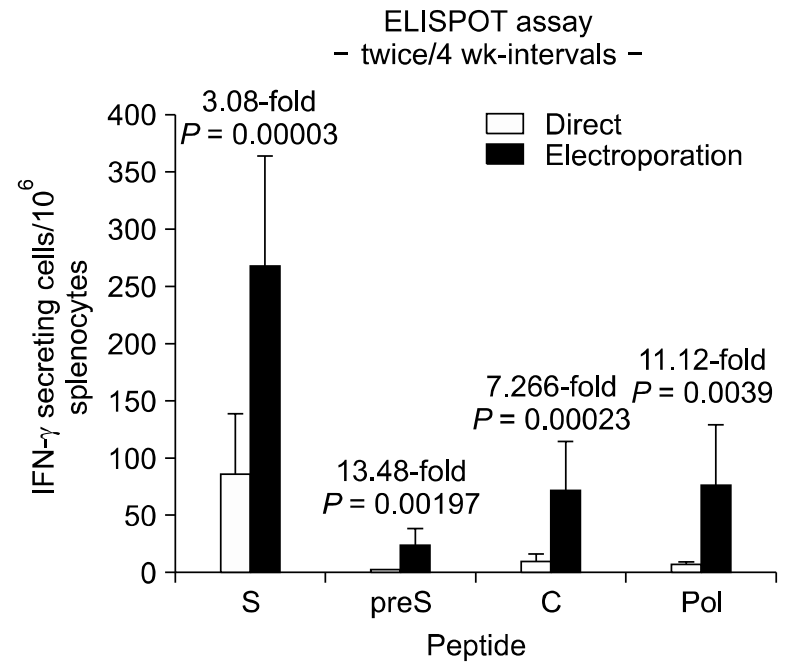

Figure 4. Cellular immune responses in protocol $1(\mathrm{~A})$ and protocol $2(\mathrm{~B})$. Cellular immune response was evaluated by counting the number of antigen-specific IFN-r secreting cells. The cellular immunity of HB-110 was enhanced after electric pulse by 1.78-fold for the S antigen and 3-fold for other antigens (pres, core, pol) in protocol 1 (3 times/2 wk-interval) (A). In the second protocol (twice/4 wk-interval), electroporation augmented the immune response by 3-fold, 13-fold, 7-fold, and 11-fold for S, preS, Core, and Pol, respectively (B). 
A

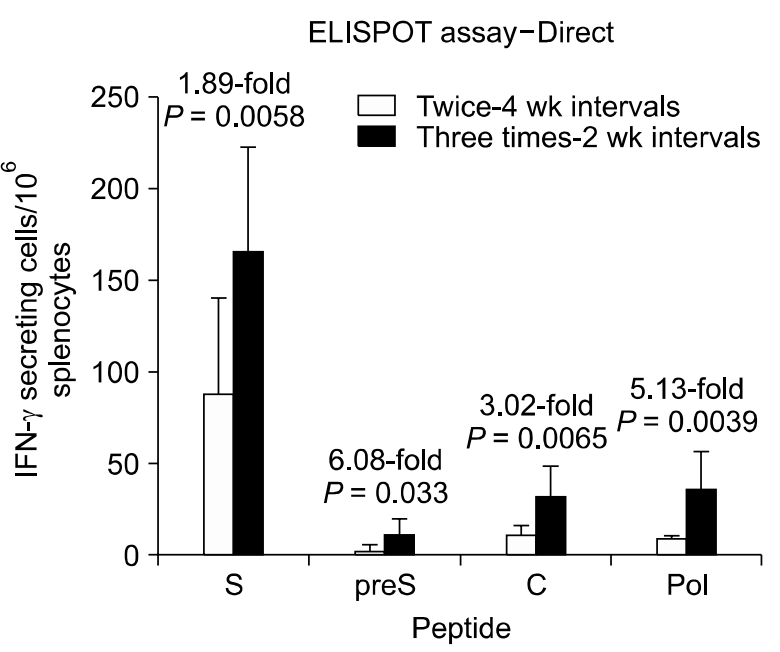

B

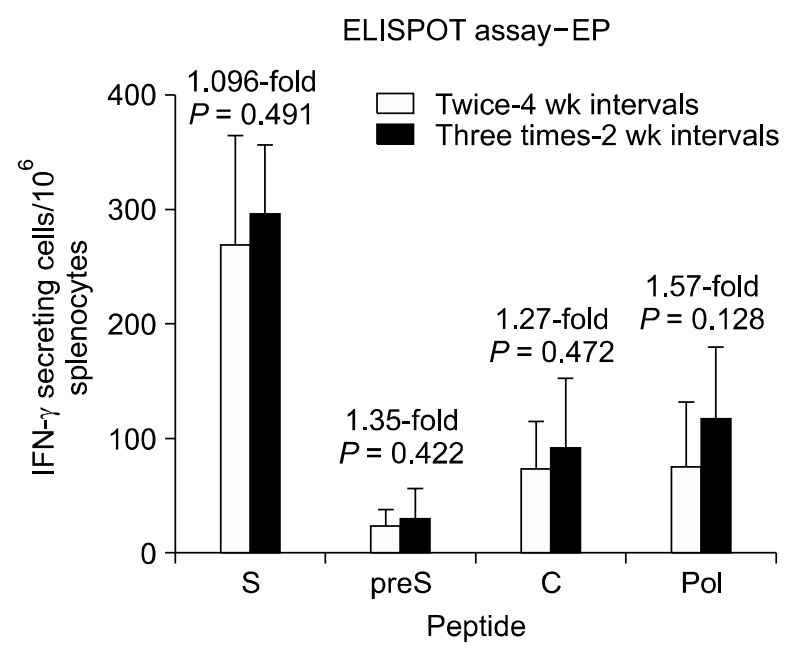

C

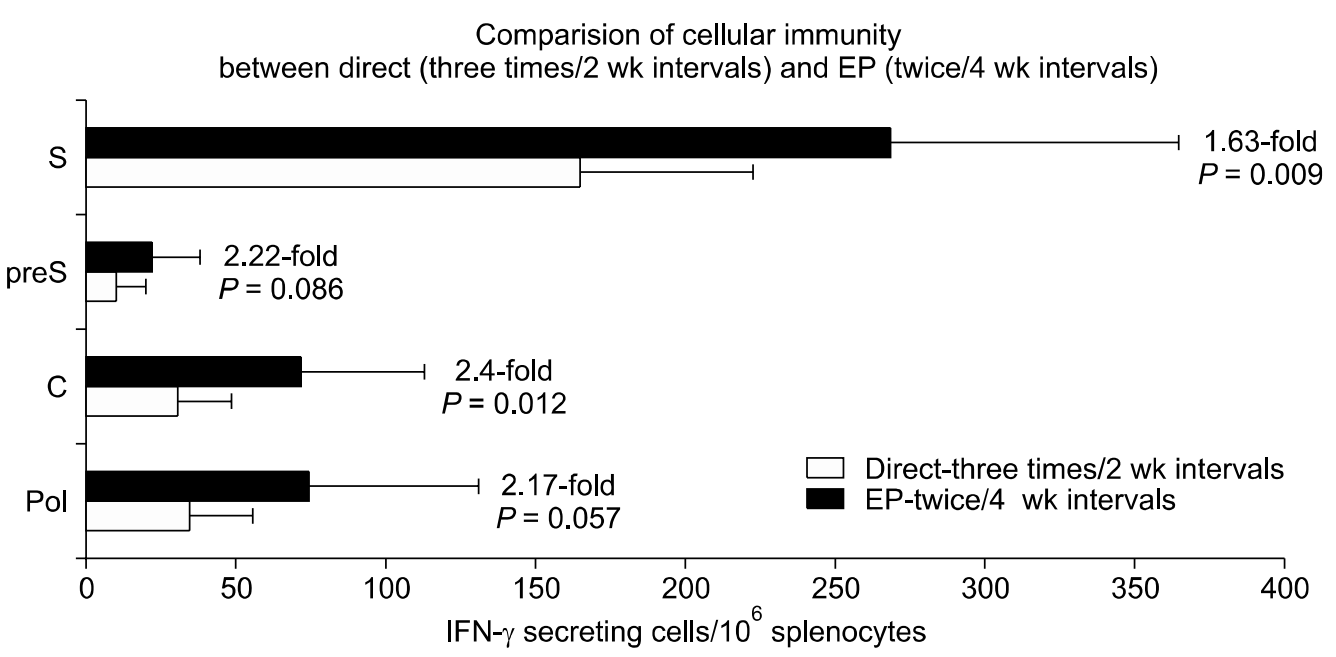

Figure 5. The differing potency of electroporation for cellular immunity in the two protocols and the overall effect on cellular immunity by electroporation. In the DI groups we noted a marked difference between protocol 1 and protocol 2, the former inducing a stronger immune response, presumably as the consequence of the increased immunization frequency (A). In the EP groups, the absolute immune response levels were similar for both protocols, thereby indicating that a high level of immune response can be achieved without frequent immunizations (B). Electroporation elicited an overall 2-fold increase in cellular immunity in the EP group of protocol 2 as compared to the DI group of protocol 1 (C).

observed in the group receiving vaccination alone (Figure 6). The anti-HBs antibody titer in the vaccine-plus-electroporation group was about 1.7fold higher than in the vaccination-alone group (data not shown).

\section{Discussion}

In this study, we observed that electroporation significantly accelerated and enhanced both the gene expression and immune responses of HB-110. Many immunological studies have empha- sized the importance of the cellular immune response for the control of viral replication. Patients recovering from acute hepatitis evidence strong polyclonal and multispecific helper and cytotoxic $T$ cell responses against the HBV protein, which is weak and undetectable in chronic patients. Animal studies have demonstrated that the elimination of HBV from hepatocytes is largely attributable to the activity of Th1 cytokines, including IFNs and TNF $\alpha$. Therefore, cellular immunity is a critical factor for the therapeutic efficacy of HB-110. In this study, we observed the potential of electroporation as a tool for improving the efficacy of HB-110. Electro- 
A

\begin{tabular}{|c|c|c|c|c|}
\hline $\mathrm{Gr}$ & $\#$ & The scheme of therapy & Route & Etc. \\
\hline 1 & $\mathrm{M} 2 / \mathrm{F} 2$ & $\mathrm{pGX10}(50 \mu \mathrm{g})$ & $\mathrm{IM}$ & \\
\hline 2 & $\mathrm{M} 3 / \mathrm{F} 3$ & $\mathrm{HB}-110(50 \mu \mathrm{g})$ & $\mathrm{IM}$ & \\
\hline 3 & $\mathrm{M} 3 / \mathrm{F} 3$ & $\mathrm{HB}-110(50 \mu \mathrm{g})$ & $\mathrm{IM} \rightarrow$ elec & \\
\hline
\end{tabular}

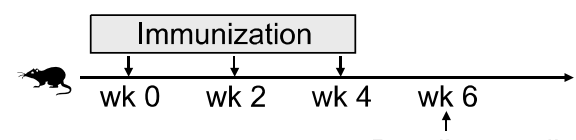

Bleeding ; antibody responses

$\&$ sacrifice ; $T$ cell responses

B

\begin{tabular}{c|c}
\hline Group & HBsAg seroconversion ${ }^{\dagger}$ rate \\
\hline pGX10 & $0 / 6$ \\
HB-110 (DI) & $1 / 6$ \\
\cline { 2 - 2 } HB-110 (EP) & $3 / 6$ \\
\hline
\end{tabular}

${ }^{\dagger} \mathrm{HBsAg}$ seroconversion means the disappearance of serum HBsAg and the induction of anti-HBs.

Figure 6. The HBsAg seroconversion rate was investigated in transgenic mice that constitutively express HBsAg in the liver. Mice were divided into three groups and given either pGX10 (vector control), HB-110 or HB-110 with electroporation. Mice were vaccinated three times at 2-week intervals and blood was collected at 2 weeks after final vaccination (A). Plasma was isolated and analyzed for HBsAg and anti-HBs antibody. In the vaccine-plus-electroporation group, three mice $(50 \%)$ showed $\mathrm{HBsAg}$ seroconversion. Only one case of seroconversion was observed in the group receiving only vaccine (B).

poration elicited an overall 2-fold increase in cellular immunity in the EP group of protocol 2 (two vaccinations/4 week intervals) as compared to the DI group of protocol 1 (three vaccinations/ 2-week intervals) (Figure $5 \mathrm{C}$ ). These results indicate that electroporation can reduce the quantity of HB-110 required for clinical efficacy by widening the intervals between vaccinations, as well as by reducing the number of vaccinations. In addition, electroporation induced a broader repertoire of cellular immune responses against subdominant antigens including pres, Core, and Pol, even though the magnitude was not as great as with the $S$ antigen. These encouraging data are consistent with previous reports regarding the potency of EP-based plasmid DNA vaccination in large animals, including monkeys (Luxembourge et al., 2005; Otten et al., 2006; Luckay et al., 2007).

The observed boost in the anti-HBs antibody response was another valuable result. At DNA doses which elicited very low responses to vaccination without $E P$, anti-HBsAg titers increased significantly and were sustained until week 12 , when EP was applied (Figure 2A). This result was quite exciting, considering that the appearance of
anti-HBs antibodies is a serological marker of complete recovery from hepatitis $B$. The potency of the humoral response elicited by EP was confirmed in a transgenic mouse model in which the HBV full genome replicates in the liver. Vaccination with EP induced $\mathrm{HBsAg}$ seroconversion in three out of six animals $(50 \%)$, whereas in the group treated with vaccine alone, only one out of six animals $(16.7 \%)$ showed seroconversion.

This preliminary study showed that electroporation is a promising tool for the delivery of $\mathrm{HB}-110$, and may enhance its immunological activities in a dosage-efficient manner. On the basis of these results, several additional studies should be conducted, including preclinical studies using disease models such as the woodchuck, and primate models such as monkeys. The effect of electroporation on the dose reduction of HB-110 should be assessed in detail in order to facilitate the design of clinical protocols.

Despite its demonstrated potency, electroporation has several problems for clinical application, including its tolerability in humans and integration issues which may possibly be caused by higher DNA uptake. There have been many efforts to develop EP devices that administer a mild electrical condition or introduce DNA in a needle-free fashion. Many reports have previously shown that the frequency of integration into chromosomal DNA after i.m. injection is negligible. Despite the increased level of DNA association with host genomic DNA, it was estimated to be at least 3 orders of magnitude below the frequency of spontaneous gene-inactivating mutations (Wang et al., 2004; Luckay et al., 2007).

HB-110, a novel therapeutic DNA vaccine against chronic hepatitis $B$, is now in phase 1 of clinical study in South Korea. We expect that the EPbased vaccination of HB-110 might exhibit more potent immunological activity, even at low doses, in future clinical studies.

\section{Acknowledgment}

This work was supported by grants from Ministry of Health and Welfare, Republic of Korea (A040013).

\section{References}

Aihara H, Miyazaki J. Gene transfer into muscle by electroporation in vivo. Nature Biotechnol 1998;16:867-70

Andre F, Mir LM. DNA electrotransfer: its principles and an updated review of its therapeutic applications. Gene Ther 2004;11(Suppl 1):S33-42

Araki K, Miyazaki JI, Hino O, Tomita N, Chisaka O, Matsubara 
K, Yamamura KI. Expression and replication of hepatitis B virus genome in transgenic mice. Proc Nati Acad Sci USA 1989;86:207-11

Calarota SA, Weiner DB. Enhancement of human immunodeficiency virus type 1-DNA vaccine potency through incorporation of T-helper 1 molecular adjuvants. Immunol Rev 2004;199:84-99

Heller LC, Heller R. In vivo electroporation for gene therapy. Hum Gene Ther 2006;7:890-7

Hurk S, Loehr BI, Babiuk LA. Immunization of livestock with DNA vaccines: current studies and future prospects. Vaccine 2001;19:2474-9

Luckay A, Sidhu MK, Kjeken R, Megati S, Chong WY, Roopchand V, Dorys GH, Abdullah R, Braun R, Montefiori DC, Rosati M, Felber BK, Pavlakis GN, Mathiessen I, Israel ZR, Eldridge JH, Egan MA. Effect of Plasmid DNA Vaccine Design and In vivo Electroporation on the resulting Vaccine-Specific Immune Responses in Resus Macaques. J Virol 2007;81:5257-69

Luxembourg A, Hannaman D, Ellefsen B, Nakamura G, Bernard R. Enhancement of immune response to an HBV DNA vaccine by electroporation. Vaccine 2006;24:4490-3

Molnar MJ, Gilbert R, Lu Y, Liu AB, Guo A, Larochelle N, Orlopp K, Lochmuller H, Petrof BJ, Nalbantoglu J, Karpati G. Factors Influencing the Efficacy, Longevity, and Safety of Electroporation-Assisted Plasmid-Based Gene Transfer into Mouse Muscles, Mol Ther 2004;10:447-55

O'Hagan DT, Singh M, Ulmer JB. Microparticles for the delivery of DNA vaccines. Immunol Rev 2004;199:191-200

Otten G, Schaefer M, Doe B, Liu H, Srivastava I, Megede J, Donnelly J, Widera G, Rabussay D, Lewis MG, Barnett S,
Ulmer JB. Enhancement of DNA vaccine potency in rhesus macaques by electroporation. Vaccine 2004;22:2489-93

Otten GR, Schaefer M, Doe B, Liu H, Megede J, Donnelly J, Rabussay D, Barnett S, Ulmer JB. Potent immunogenecity fo an HIV-1 gag-pol fusion DNA vaccine delivered by in vivo electroporation. Vaccine 2006;24:4503-9

Penga B, Zhaoa Y, Xub L, Xua Y. Electric pulses applied prior to intramuscular DNA vaccination greatly improve the vaccine immunogenicity. Vaccine 2007;25:2064-73

Selby M, Goldbeck C, Pertile T, Walsh R, Ulmer J. Enhancement of DNA vaccine potency by electorporation in vivo. J Biotech 2000;83:147-52

Tjelle TE, Salte R, Mathiesen I, Kjeken R. A novel electorporation device for gene delivery in large animals and humans. Vaccine 2006;24:4667-70

Wang XD, Tang JG, Xie XL, Yang JC, Li S, Ji JG, Gu J. A comprehensive study of optimal conditions for naked plasmid DNA transfer into skeletal muscle by electroporation. J Gene Med 2005;7:1235-45

Wang Z, Troilo PJ, Wang X, Griffiths TG, Pacchione SJ, Barnum AB, Harper, LB, Pauley CJ, Niu Z, Denisova L, Follmer TT, Rizzuto G, Ciliberto G, Fattori E, Monica NL, Manam S and Ledwith BJ. Detection of integration of plasmid DNA into host genomic DNA following intramuscular injection and electroporation. Gene Ther 2004;11:711-21

Yang SH, Lee CG, Park SH, Im SJ, Kim YM, Son JM, Wang JS, Yoon SK, Song MK, Ambrozaitis A, Kharchenko N, Yun YD, Kim CM, Kim CY, Lee SH, Kim BM, Kim WB, Sung YC. Correlation of antiviral T-cell responses with suppression of viral rebound in chronic hepatitis B carriers: a proof-ofconcept study. Gene Ther 2006;1-8 